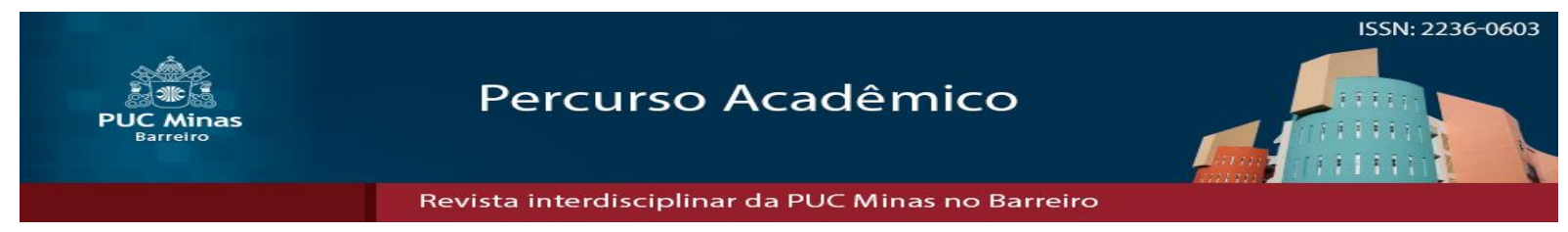

\title{
Ações e serviços em HIV/AIDS de Porto Alegre: análise dos Relatórios de Gestão 2010-2014
}

\author{
Porto Alegre actions and services on HIV/AIDS: \\ analysis of Management Reports 2010-2014
}

\author{
Priscila Alessandra Janke Dutra ${ }^{1}$ \\ Vania Roseli Correa de Mello ${ }^{2}$ \\ Daniel Dall'Igna Ecker ${ }^{3}$
}

\section{Resumo}

$\mathrm{O}$ artigo discute as ações e serviços de atenção especializada direcionados ao enfrentamento de HIV/AIDS desenvolvidos pela Secretaria Municipal da Saúde de Porto Alegre, Rio Grande do Sul, Brasil (SMS/PMPA). Para isso, analisa os Relatórios Anuais de Gestão no período de 2010 a 2014. Em conjunto com a UNAIDS (Programa das Nações Unidas para o Combate à AIDS), a SMS/PMPA desenvolveu um plano integrado de ações que articula gestores, sociedade civil e agências internacionais de controle do HIV/AIDS em um esforço coletivo para modificar o panorama atual dos casos na cidade de Porto Alegre. Essas ações visavam reforçar o compromisso político de atender às necessidades dos usuários, mobilizando comunidades para efetivamente exigir mudanças transformadoras, bem como direcionar recursos para a realização das intervenções necessárias. Os resultados permitem observar que as ações e serviços de saúde, com exceção das consultas médicas, apresentaram uma elevação, resultando na diminuição de $16,2 \%$ nas taxas de incidência e de $17 \%$ nos coeficientes de mortalidade da doença no município. Corroborado pelos dados, ressalta-se a importância das ações e serviços já existentes para que se garanta o acompanhamento e a qualidade da assistência aos portadores do vírus, assim como, o controle e diminuição dos casos de HIV/AIDS.

Palavras-chave: HIV. AIDS. Saúde Pública. Relatório Anual de Gestão. Porto Alegre.

\footnotetext{
Artigo recebido em 17 de Setembro de 2018 e aprovado em 14 de Dezembro de 2018

${ }^{1}$ Graduada em Administração, com ênfase em Sistemas e Serviços de Saúde, pela Universidade Estadual do Rio Grande do Sul (UERGS). Atualmente trabalha no Serviço de Hospitalidade do Hospital de Clínicas de Porto Alegre (HCPA-UFRGS). Brasil. E-mail: dutrapriscila41@ gmail.com

${ }^{2}$ Doutora em Psicologia pela Universidade Federal Fluminense (UFF), Mestre em Psicologia Social e Institucional pela Universidade Federal do Rio Grande do Sul (UFRGS), Especialização em Saúde Pública pela Escola de Saúde Pública (ESP) do Rio Grande do Sul,1 Especialização em Docência na Saúde pela Universidade Federal do Rio Grande do Sul (UFRGS) e Graduação em Psicologia pela Pontifícia Universidade Católica do Rio Grande do Sul (PUCRS). Servidora Pública na Prefeitura Municipal de Porto Alegre (PMPA) na Secretaria de Estado da Saúde do Rio Grande do Sul. Atualmente é Professora Adjunta, área de Saúde Coletiva, na Universidade Estadual do Rio Grande do Sul (UFRGS). Brasil. E-mail: vaniarcmello@gmail.com

${ }^{3}$ Doutorando em Psicologia Social e Institucional (bolsista CAPES) na UFRGS, realizando período sanduíche na Universidade Federal de Santa Catarina - UFSC, Mestre em Psicologia Social e Institucional (UFRGS) com período sanduíche na Universidade Federal de Alagoas (UFAL). Graduado pela Pontifícia Universidade Católica do Rio Grande do Sul (PUCRS). Experiência como bolsista no Programa de Educação Tutorial (PET/MEC), bolsista de Iniciação Científica (CNPq) no Programa de Pós-Graduação em Psicologia Social e Institucional da Universidade Federal do Rio Grande do Sul UFRGS trabalhando em atividades de ensino, projetos de pesquisa e de extensão. Filiado à - Associação Brasileira de Psicologia Social (ABRAPSO) trabalhou na Secretaria Administrativa, junto à Direção Nacional, no mesmo período. Atualmente é Editor-Executivo na Revista Eletrônica Científica da e Orientador de alunas (os) de graduação da Universidade Estadual do Rio Grande do Sul (UERGS) na área das Ciências Humanas. Brasil. E-mail: daniel.ecker@ hotmail.com
}

Percurso Acadêmico, Belo Horizonte, v. 8, n. 16, jul./dez. 2018

Página 242 


\begin{abstract}
The article discusses the actions and services of specialized attention directed to the confrontation of HIV/AIDS developed by the Municipal Health Department of Porto Alegre, Rio Grande do Sul, Brazil (SMS/PMPA). To this end, it reviews the Annual Management Reports from 2010 to 2014. In conjunction with UNAIDS, the SMS/PMPA has developed an integrated action plan that articulates managers, civil society and international agencies to control HIV/AIDS in a collective effort to modify the current panorama of cases in the city of Porto Alegre. These actions aimed at reinforcing the political commitment to meet the needs of users, mobilizing communities to effectively demand transformative changes, as well as directing resources for the necessary interventions. The results show that health actions and services, with the exception of medical consultations, showed a rise, resulting in a $16.2 \%$ decrease in incidence rates and a $17 \%$ decrease in the mortality coefficients of the disease in the municipality. Corroborated by the data, it is important to highlight the importance of existing actions and services in order to guarantee the follow-up and quality of care for the virus carriers, as well as the control and reduction of HIV/AIDS cases.
\end{abstract}

Key words: HIV. AIDS. Public health. Annual Management Report. Porto Alegre.

\title{
1 Introdução
}

Os altos índices de contágio por HIV permanecem como um dos grandes desafios para a saúde pública mundial. Dados da Coordenação das Nações Unidas no Brasil, de 2010, afirmam que aproximadamente 33 milhões de pessoas vivem com HIV em todo o mundo. Em estudo elaborado pelo UNAIDS, aponta-se que, apesar dos inúmeros avanços já registrados, as regiões mais pobres do planeta seguem sendo as mais afetadas na guerra contra a AIDS. Sem alimentação e assistência médica adequadas, geralmente disponíveis em países desenvolvidos, uma grande quantidade de pessoas sofre e morre de complicações relacionadas ao HIV no mundo, afetando também o crescimento econômico dos países, ao reduzir a disponibilidade de capital humano (PROGRAMA CONJUNTO DAS NAÇÕES UNIDAS SOBRE HIV/AIDS, 2010).

No Brasil, o acesso ao tratamento de HIV/AIDS é universal e gratuito pelo Sistema Único de Saúde (SUS). Toda pessoa infectada tem direito a ser acompanhada na rede de saúde e, sempre que necessário, iniciar o tratamento com a medicação antirretroviral. Estima-se que, atualmente, 630.000 pessoas de 15 a 49 anos vivam com HIV no Brasil. Dessas, em torno de 255.000 não sabem sua condição sorológica. A cobertura de teste de HIV na população brasileira passou de $28 \%$ em 2004 para $37 \%$ em 2008, sendo maior entre as mulheres por causa da oferta da testagem durante o prénatal. A oferta de teste de HIV pelo SUS passou de 3,3 milhões em 2003 para 5,1 milhões de unidades em 2011 (BRASIL, 2012a). 
A resposta brasileira ao HIV é destaque no cenário internacional e resulta de ações compartilhadas entre o governo federal, estados e municípios, com investimentos em prevenção e vigilância que ultrapassam mais de $\mathrm{R} \$ 400$ milhões, por ano. Em 2011, houve um aumento de $91 \%$ no valor destinado à política de controle do vírus, passando de $\mathrm{R} \$ 68$ milhões, em 2002, para $\mathrm{R} \$ 130$ milhões investidos (BRASIL, 2012a). A taxa de incidência nacional de HIV em 2012 foi de 20,2 casos para cada 100.000 habitantes. No Rio Grande do Sul essa taxa permanece acima da média nacional, ficando em 41,4 casos para cada 100.000 habitantes (BRASIL, 2012a).

O município de Porto Alegre, capital do Estado do Rio Grande do Sul (RS), conta com uma taxa de 99,8 casos a cada 100 mil habitantes, bem acima da média estadual. O Boletim Epidemiológico número 51 da Coordenadoria Geral de Vigilância em Saúde (CGVS) da Secretaria Municipal da Saúde de Porto Alegre (SMS/PMPA) de maio de 2013, mostra que a maior taxa de incidência está na Gerência Distrital Partenon/Lomba do Pinheiro com 137,01 casos diagnosticados de HIV a cada 100 mil habitantes no ano de 2012. Esses dados demonstram a complexidade e a transversalidade da epidemia, que demanda a mobilização de vários setores e parceiros, por meio de uma abordagem multissetorial e integrada que oferte respostas efetivas às demandas cada vez mais crescentes e significativas que se apresentam à rede de atenção à saúde do portador de HIV/AIDS e que devem ser desenvolvidas para enfrentar a elevação das taxas de incidência no Brasil (PROGRAMA CONJUNTO DAS NAÇÕES UNIDAS SOBRE HIV/AIDS, 2010).

O HIV está entre os temas prioritários do Sistema Único de Saúde e, ao mesmo tempo, os dados epidemiológicos apontam para as altas taxas de incidência, em especial, na capital do Estado do RS. Diante do contexto apresentado, este artigo se ocupa da seguinte questão: de que modo as ações e serviços de saúde direcionadas ao enfrentamento de HIV/AIDS estão repercutindo na redução dos casos da doença no município de Porto Alegre? Para tanto, este artigo tem como objetivo analisar as ações e serviços de atenção especializada direcionados ao enfrentamento de HIV/AIDS, desenvolvidos pela Secretaria Municipal da Saúde de Porto Alegre, por meio da análise dos Relatórios Anuais de Gestão da SMS/PMPA no período de 2010 a 2014. 


\section{HIV/AIDS no cenário nacional}

O primeiro caso de HIV no Brasil foi relatado em 1982 e, em 1986, foi criado pelo Ministério da Saúde, o Programa Brasileiro de DST/AIDS (PNDST/AIDS), com a responsabilidade de estabelecer um plano nacional para o enfrentamento da epidemia no país. O programa foi reestruturado em 1992, com maior atenção na articulação entre o governo e as organizações não governamentais (LEVI; VITÓRIA, 2002). Em 1996, o Congresso Nacional, apoiado e pressionado pela sociedade civil organizada, promulgou a Lei $\mathrm{n}^{\circ} 9.313$ de 13 de novembro de 1996 (BRASIL, 1996) que tornou obrigatória a provisão de acesso universal sem custo adicional à terapia antirretroviral para todos os portadores de HIV que dela necessitem. Em 2013, a estimativa era que 734 mil pessoas estavam vivendo com o HIV em território nacional. Destas, 589 mil (80\%) sabiam de seu diagnóstico positivo. Entre esses diagnosticados, 355 mil pessoas (60\%) estavam em tratamento com antirretrovirais e 83\% (293 mil pessoas) com a carga viral indetectável. Em 2014, mais 49 mil pessoas iniciaram tratamento para a infecção (BRASIL, 2014).

De acordo com registros do SINAN (Sistema de Informação de Agravos de Notificação), a incidência do HIV, no período de 2007 a 2017, no que se refere às faixas etárias, identificou que a maioria dos casos de infecção pelo HIV encontra-se nas faixas de 20 a 34 anos, com percentual de 52,5\% dos casos. Segundo sexo, foi notificado um total de $131.969(67,9 \%)$ casos em homens e 62.198 (32,1\%) casos em mulheres. Relacionado à raça/cor da pele autodeclarada, 47,6\% são entre brancos e 51,5\% entre pretos e pardos. No sexo masculino, 49,6\% dos casos estão entre brancos e 49,4\% entre pretos e pardos; entre as mulheres, 43,2\% dos casos são entre brancas e 55,9\% entre pretas e pardas (BRASIL, 2017). Dentre as populações mais vulneráveis, entre 2009 e 2010, as prevalências eram: usuários de drogas (prevalência de 5,91\%), homens que fazem sexo com outros homens - HSH (10,5\%) e profissionais do sexo feminino $(4,9 \%)$. Nos últimos 12 anos, no Brasil, a taxa de incidência baixou de 7,6 para 6,3 a cada 100 mil pessoas representando uma queda de 17\%. Estes dados não são uniformes considerando as diferenças regionais do nosso país (BRASIL, 2012a).

Segundo o Departamento de DST, AIDS e Hepatites Virais, do total de casos registrados entre 1980 e junho de 2013, 379.045 (55,2\%) está na Região Sudeste; 137.126 (20,0\%) na Região Sul; 95.516 (13,9\%) na Região Nordeste; $39.691(5,8 \%)$ na 
Região Centro-Oeste; e $35.100(5,1 \%)$ na Região Norte, de acordo com o gráfico abaixo:

\section{Gráfico 1 - Casos registrados de HIV/AIDS no Brasil entre 1980 e 2013.}

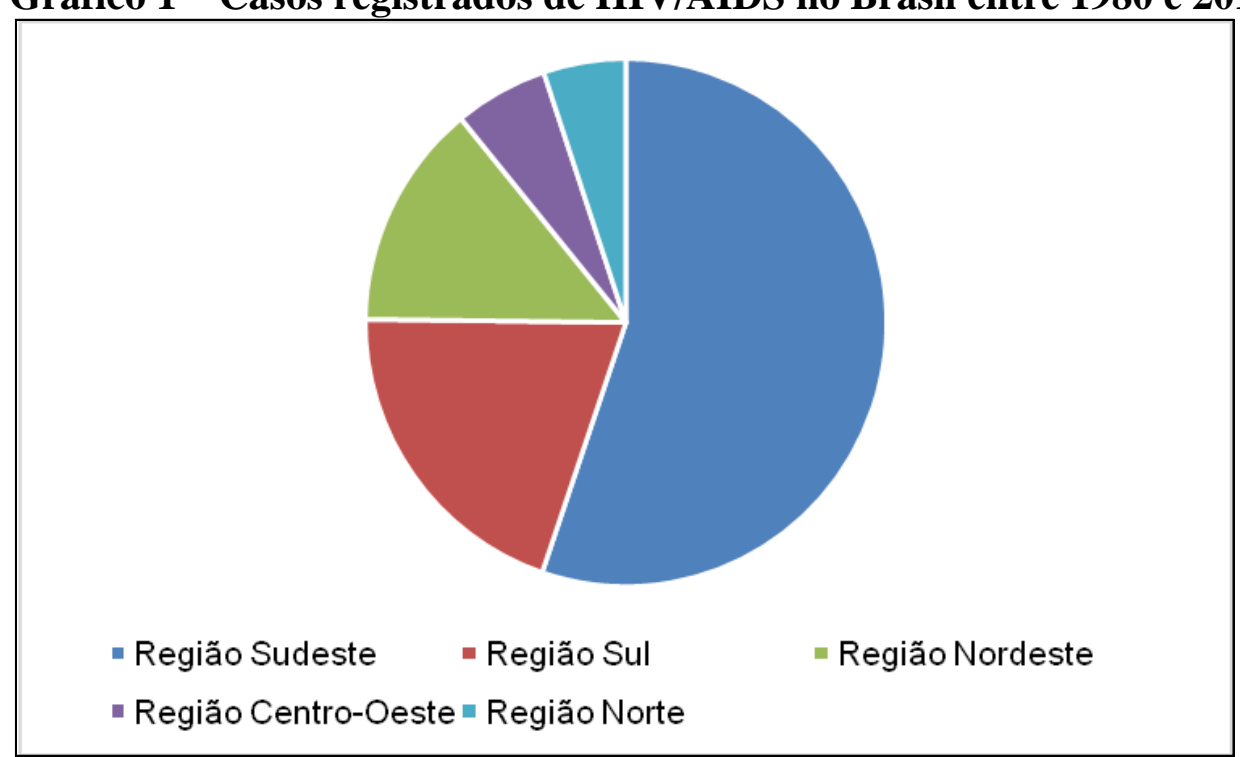

Fonte: BRASIL, 2013.

Na última década, a taxa de detecção da HIV no Brasil sofreu elevação de $2 \%$, com diferenças significativas entre as regiões, sendo que na região sul observou-se uma taxa de $0,3 \%$ (RIO GRANDE DO SUL, 2014).

\subsection{HIV/AIDS no município de Porto Alegre/RS}

A manutenção de taxas elevadas na incidência de casos de HIV tem chamado atenção para a epidemia da doença na cidade na última década. Os primeiros casos no município reportam ao início dos anos 80 e o total acumulado de casos de 1983 até 31 de maio de 2013 é de 24.299 casos de HIV, sendo 96,19\% em adultos e 3,81\% em menores de 13 anos. Sem contar os óbitos, existem atualmente 14.100 pessoas portadoras de HIV no município. Estima-se que 28.187 pessoas vivam com HIV em Porto Alegre atualmente, o que corresponde a $2 \%$ da população da capital gaúcha (PORTO ALEGRE, 2013). 


\section{Quadro 1 - Incidência de HIV/AIDS no município de Porto Alegre/RS}

\begin{tabular}{|c|c|}
\hline ANO & $\begin{array}{c}\text { INCIDÊNCIA DE HIV/AIDS por 100.000 } \\
\text { habitantes }\end{array}$ \\
\hline 2007 & 91,46 \\
\hline 2008 & 99,98 \\
\hline 2009 & 95,67 \\
\hline 2010 & 103,45 \\
\hline 2011 & 94,01 \\
\hline 2012 & 92,52 \\
\hline & Fonte: PORTO ALEGRE, 2013.
\end{tabular}

Podemos observar que as variações nas taxas de incidência de HIV/AIDS no município de Porto Alegre apresentam uma média de aproximadamente 96,18 casos de HIV a cada 100.000 no período demonstrado. Após o ápice, em 2010, de 103,45 casos a cada 100.000 habitantes, podemos observar a tendência de um declínio nas taxas de incidência nos anos seguintes de forma geral. Em Porto Alegre, os serviços de saúde estão distribuídos nos territórios dos 17 Distritos Sanitários: Ilhas, Humaitá/Navegantes, Centro, Noroeste, Norte, Eixo Baltazar, Leste, Nordeste, Glória, Cruzeiro, Cristal, Sul, Centro-Sul, Partenon, Lomba do Pinheiro, Restinga e Extremo-Sul. Os distritos sanitários formam as Gerências Distritais, estruturas administrativas e gestoras da saúde no município, distribuídas em oito regiões de saúde, como podemos observar na Figura 2 a seguir: 
Figura 2 - Áreas de abrangência das oito Gerências Distritais (GD) de saúde do município de Porto Alegre/RS, 2011.

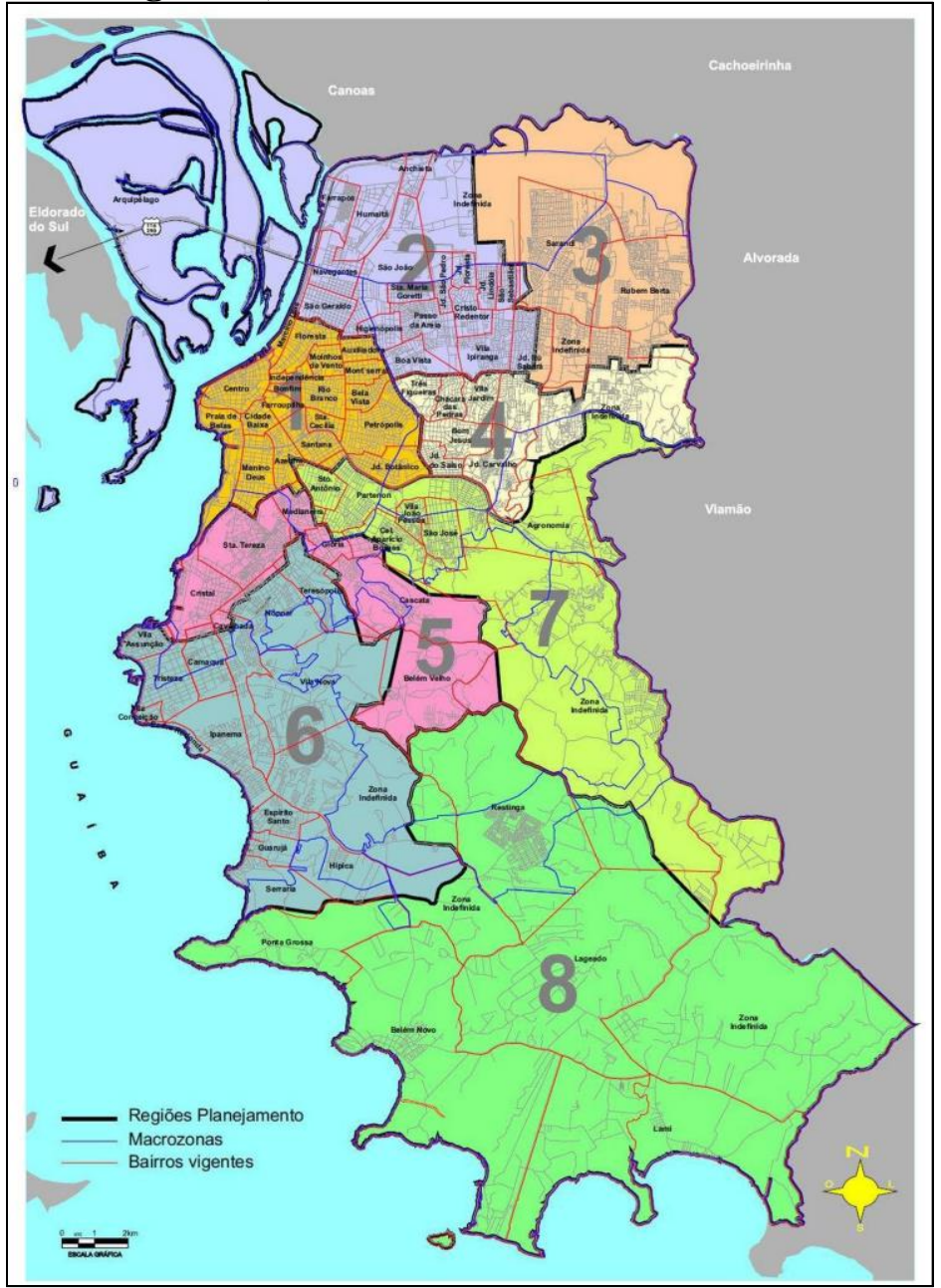

Fonte: Adaptado de Prefeitura Municipal de Porto Alegre/RS, Secretaria do Planejamento Municipal. 1) Centro; 2) Noroeste/Humaitá/Navegantes/Ilhas; 3) Norte/Eixo Baltazar; 4) Leste /Nordeste; 5) Glória/Cruzeiro/Cristal; 6) Sul/Centro-Sul; 7) Partenon/Lomba do Pinheiro; 8) Restinga/Extremo-Sul (PORTO ALEGRE, 2018).

Segundo o site da Secretaria Municipal de Saúde (SMS), da cidade de Porto Alegre, RS, as Gerências Distritais são compostas por Unidades de Saúde, Centros de Especialidades e Serviços Especializados Ambulatoriais e Substitutivos. Nos territórios dos DS e das GD também estão os Pronto-Atendimentos (PAs), as Bases do SAMU e os hospitais gerais e especializados próprios e conveniados ao SUS, formando a rede de serviços do SUS em Porto Alegre (PORTO ALEGRE, 2018). Dados divulgados pela SMS informam que na distribuição das incidências de HIV/AIDS por Gerência Distrital de Saúde, os distritos do Partenon e Lomba do Pinheiro (GD PLP) apresentam as taxas mais elevadas, seguido pelos distritos da GD Leste e Nordeste (GD LENO) e Restinga (GD RES). Essas três Gerências Distritais possuem taxas maiores que a da cidade de 
Porto Alegre, acima de 100 casos para cada 100.000 habitantes, principalmente nos anos de 2011 e 2012, como podemos observar no gráfico a seguir:

Gráfico 2 - Coeficiente de incidência de AIDS por GD de saúde em Porto Alegre/RS, 2007 a 2012.

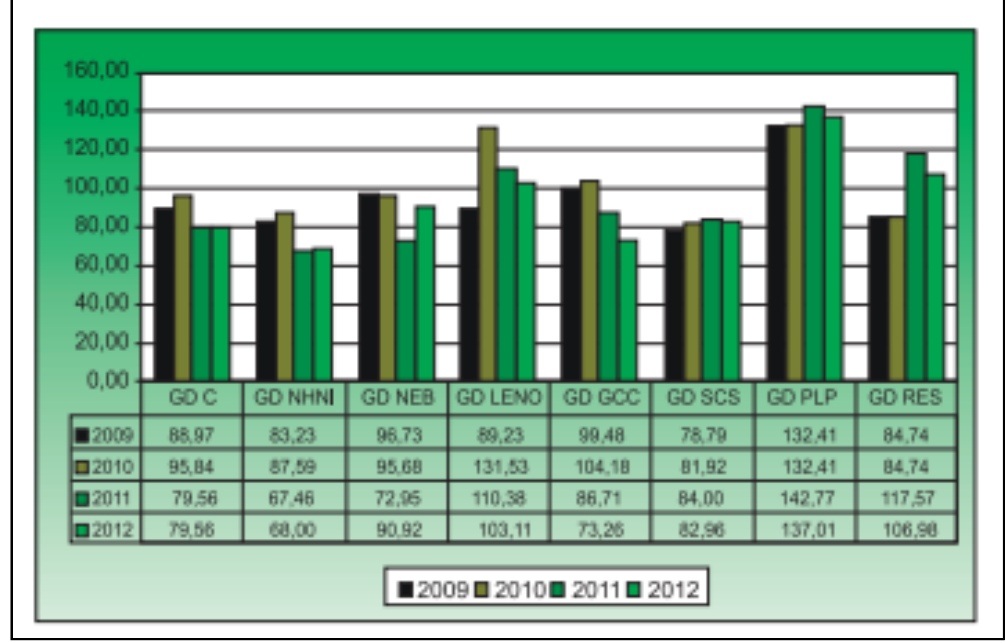

Fonte: PORTO ALEGRE, 2013.

De acordo com o Plano Municipal de Saúde para os anos de 2010 a 2013, desenvolvido pela SMS/PMPA, as áreas que correspondem às Gerências Distritais Partenon/Lomba do Pinheiro e Restinga, apresentam vulnerabilidades socioeconômicas que se destacam das demais regiões (BRASIL, 2000). A Gerência Distrital Partenon/Lomba do Pinheiro abrange um grande espaço geográfico situada na Zona Leste do Município com, aproximadamente, 181 mil habitantes (PORTO ALEGRE, 2015). Inclui mais de sessenta vilas e sete bairros, com diferentes níveis de urbanização e infraestrutura, cuja população apresenta-se, em grande parte, em condição de pobreza ou indigência (PORTO ALEGRE, 2010).

Esses dados corroboram a necessidade de pesquisas na área que visem implementar, avaliar e construir políticas públicas dedicadas ao enfrentamento do HIV/AIDS no município de Porto Alegre. Seguindo a proposta de planos integrados, que apontam a necessidade de articulação entre diversos atores da sociedade, compreende-se que se possa abordar a incidência do vírus de forma diferenciada, exigindo um esforço coletivo de gestores, sociedade civil e agências internacionais de controle da epidemia do HIV/AIDS no sentido de incidir sobre o panorama atual (PORTO ALEGRE, 2012). 


\section{Procedimentos metodológicos}

Este artigo resulta de uma pesquisa que consistiu num estudo de abordagem mista, que articulou dados e relações quantitativas com posterior análise qualitativa, desenvolvida a partir de dados secundários obtidos por meio de pesquisa documental de domínio público. Dados referentes ao ano de 1982 a 2017 foram utilizados para contextualizar a pesquisa, compondo a revisão teórica, mas não sendo o objetivo da pesquisa em si. Para a pesquisa, fizemos o uso dos Relatórios de Gestão de 2010 até 2014 por serem os Relatórios que apresentavam, de forma completa, todos os indicadores desejados ao estudo.

Por ser uma pesquisa quantitativa, segundo Richardson (1989), caracteriza-se pelo emprego da quantificação, na coleta de informações e no tratamento dessas, através de técnicas estatísticas simples e complexas. Entre os tipos de pesquisas quantitativas, segundo Diehl (2004) pode-se citar os de correlação de variáveis ou descritivos (os quais por meio de técnicas estatísticas procuram explicar seu grau de relação e o modo como estão operando), os estudos comparativos causais (onde o pesquisador parte dos efeitos observados para descobrir seus antecedentes), e os estudos experimentais (que proporcionam meios para testar hipóteses). Pode-se definir a relação entre pesquisa quantitativa e qualitativa da seguinte forma:

Diferentemente da pesquisa qualitativa, os resultados da pesquisa quantitativa podem ser quantificados. Como as amostras geralmente são grandes e consideradas representativas da população, os resultados são tomados como se constituíssem um retrato real de toda a população alvo da pesquisa. A pesquisa quantitativa se centra na objetividade. Influenciada pelo positivismo, considera que a realidade só pode ser compreendida com base na análise de dados brutos, recolhidos com o auxílio de instrumentos padronizados e neutros. A pesquisa quantitativa recorre à linguagem matemática para descrever as causas de um fenômeno, as relações entre variáveis, etc. A utilização conjunta da pesquisa qualitativa e quantitativa permite recolher mais informações do que se poderia conseguir isoladamente (FONSECA, 2002, p. 20).

A pesquisa qualitativa, de acordo com Minayo (2001), trabalha com o universo de significados, motivos, aspirações, crenças, valores e atitudes, o que corresponde a um espaço mais profundo das relações, dos processos e dos fenômenos que não podem ser reduzidos à operacionalização de variáveis. Mayring (2002) apresenta seis delineamentos da pesquisa qualitativa: estudo de caso, análise de documentos, pesquisa- 
ação, pesquisa de campo, experimento qualitativo e avaliação qualitativa. Caracteriza-se como um estudo de caso que de acordo com Gil (1991), consiste no estudo em profundidade de poucos objetos, de forma a permitir conhecimento amplo e específico do mesmo sem buscar generalizações sobre o tema.

Um estudo de caso pode ser caracterizado como um estudo de uma entidade bem definida como um programa, uma instituição, um sistema educativo, uma pessoa, ou uma unidade social. Visa conhecer em profundidade o como e o porquê de uma determinada situação que se supõe ser única em muitos aspectos, procurando descobrir o que há nela de mais essencial e característico. $O$ pesquisador não pretende intervir sobre o objeto a ser estudado, mas revelá-lo tal como ele o percebe. O estudo de caso pode decorrer de acordo com uma perspectiva interpretativa, que procura compreender como é o mundo do ponto de vista dos participantes, ou uma perspectiva pragmática, que visa simplesmente apresentar uma perspectiva global, tanto quanto possível completa e coerente, do objeto de estudo do ponto de vista do investigador (FONSECA, 2002, p. 33).

Para Yin (2001), estudo de caso é uma investigação empírica, um método que abrange desde planejamento, técnicas de coleta de dados a análise dos mesmos. No contexto de um estudo de caso, delimitado como a coleta e análise de dados sobre um exemplo individual para definir um fenômeno mais amplo (VOGT, 1993) podem-se coletar e analisar tanto dados quantitativos quanto qualitativos. Nos estudos em atenção à saúde conceitua-se o estudo de caso como:

Os estudos de casos podem ser desenvolvidos de diferentes maneiras, mas possuem diversos aspectos em comum. Começam com a identificação de perguntas de pesquisa que emergem de preocupações a respeito das implicações de novas políticas ou, às vezes de afirmativas sobre novas teorias de gerenciamento. Em geral os estudos de caso são desenvolvidos prospectivamente, examinando a implementação de políticas em um período de tempo (POPE; MAYS, 2009, p. 129).

Deste modo, os Relatórios de Gestão estudados foram selecionados e analisados ano a ano, buscando traçar um panorama das informações disponibilizadas a respeito do HIV/AIDS, a partir das quais foram elaborados quadros e gráficos. Os dados numéricos contendo os indicadores das ações e serviços foram alocados em categorias por meio de quadros, trazendo dados de cada ano do período selecionado. Os indicadores apresentados são: consultas médicas (disponibilizadas, agendadas e realizadas); consultas de enfermagem e atendimentos de Serviço Social realizados; distribuição de 
preservativos (masculinos e femininos) e gel lubrificante. Dados relativos às taxas de incidência e ao coeficiente de mortalidade foram organizados em quadros, ano a ano, para na sequência, serem elaborados gráficos que visam apresentar a relação entre os mesmos no período. A incidência de uma doença, em um determinado local e período, é o número de casos novos da doença que iniciaram no mesmo local e período. Traz a ideia de intensidade com que acontece uma doença numa população, mede a frequência ou probabilidade de ocorrência de casos novos de doença na população. Se mede pela seguinte formula: $\mathrm{N}^{\mathrm{o}}$ de casos novos de determinada doença em dado local e período $\mathrm{x}$ 10n / População do mesmo local e período. Já a mortalidade, representa o risco ou probabilidade que qualquer pessoa na população apresenta de poder vir a morrer ou de morrer em decorrência de uma determinada doença. Se mede pela seguinte formula: $\mathrm{N}^{\mathbf{o}}$ de óbitos por determinada causa (ou grupo de causas), no período x 100.000/ População na metade do período (PEREIRA, 2004).

No que se refere aos princípios éticos, seguindo orientações do documento "Perguntas e Respostas" elaborado pela Comissão Nacional de Ética em Pesquisa (CONEP), as pesquisas que envolvem apenas dados de domínio público, não identificando os participantes da pesquisa, ou pesquisas estritamente de revisão bibliográfica, sem o envolvimento de seres humanos, não necessitam de aprovação por parte do Sistema CEP/CONEP (BRASIL, 2018).

\section{Resultados}

\subsection{Relatórios de Gestão de 2010 a 2014}

As ações e serviços de atenção especializada direcionados ao enfrentamento de HIV/AIDS em Porto Alegre desenvolvidos pela SMS/PMPA, presentes nos Relatórios de Gestão do período selecionado, incluem a totalidade da produção conjunta dos Serviços de Assistência Especializados (SAE) do Centro de Saúde da Vila dos Comerciários (CSVC) e Centro de Saúde do IAPI, que são as referências no município para o atendimento aos portadores de HIV/AIDS. A partir do ano de 2014, o Relatório de Gestão agregou os dados referentes ao Serviço de Assistência Especializada do Centro de Saúde Santa Marta. Devido às modificações ocorridas a partir do ano de 2012 na apresentação das informações constantes nos Relatórios de Gestão, estas foram sistematizadas em quadros elaborados pela autora, ano a ano, apresentando as ações e 
serviços e seus indicadores. As taxas de incidência de HIV/AIDS foram analisadas por meio dos Boletins Epidemiológicos disponíveis no site da SMS/PMPA.

\subsection{Aspectos quantitativos das ações e serviços em HIV/AIDS no município de Porto Alegre/RS.}

Gráfico 3 - Evolução das consultas médicas disponibilizadas e realizadas.

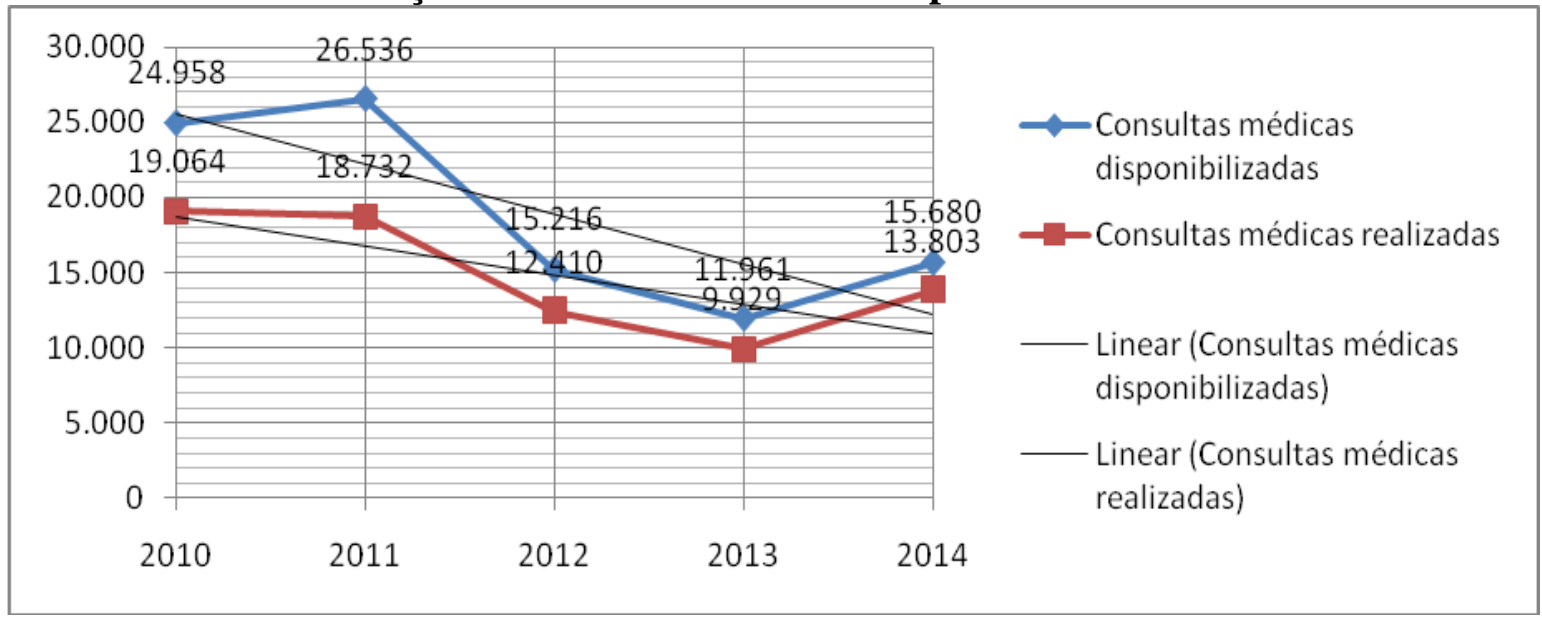

Fonte: autores.

A análise das informações disponíveis nos Relatórios de Gestão (2010-2014) nos permite observar que houve um aumento de aproximadamente $6,32 \%$ nas consultas médicas disponibilizadas entre os anos de 2010 a 2011. No entanto, no mesmo período, tivemos um decréscimo de aproximadamente $1,74 \%$ no número de consultas médicas realizadas. A partir de 2012 tanto as consultas médicas ofertadas quanto às realizadas entraram em declínio. Segundo o Relatório de Gestão de 2012, esta redução ocorreu pela diminuição do quadro efetivo de profissionais e também devido à participação em congressos e licenças saúde. No ano de 2014 a oferta de consultas médicas retoma seu crescimento em decorrência da contratação de novos profissionais, do aumento da carga horária dos médicos e, especialmente, em decorrência da implantação do novo serviço de atendimento especializado em HIV/AIDS no município, o SAE Santa Marta (PORTO ALEGRE, 2014).

Ao acompanharmos a evolução das consultas médicas realizadas ao longo do período de 2010 à 2014, verificamos um decréscimo de aproximadamente 27,6\% nas mesmas. Quanto as consultas médicas disponibilizadas, observa-se um declínio de aproximadamente $37,18 \%$ no período: 


\section{Gráfico 4 - Evolução das consultas de enfermagem e atendimentos do serviço} social

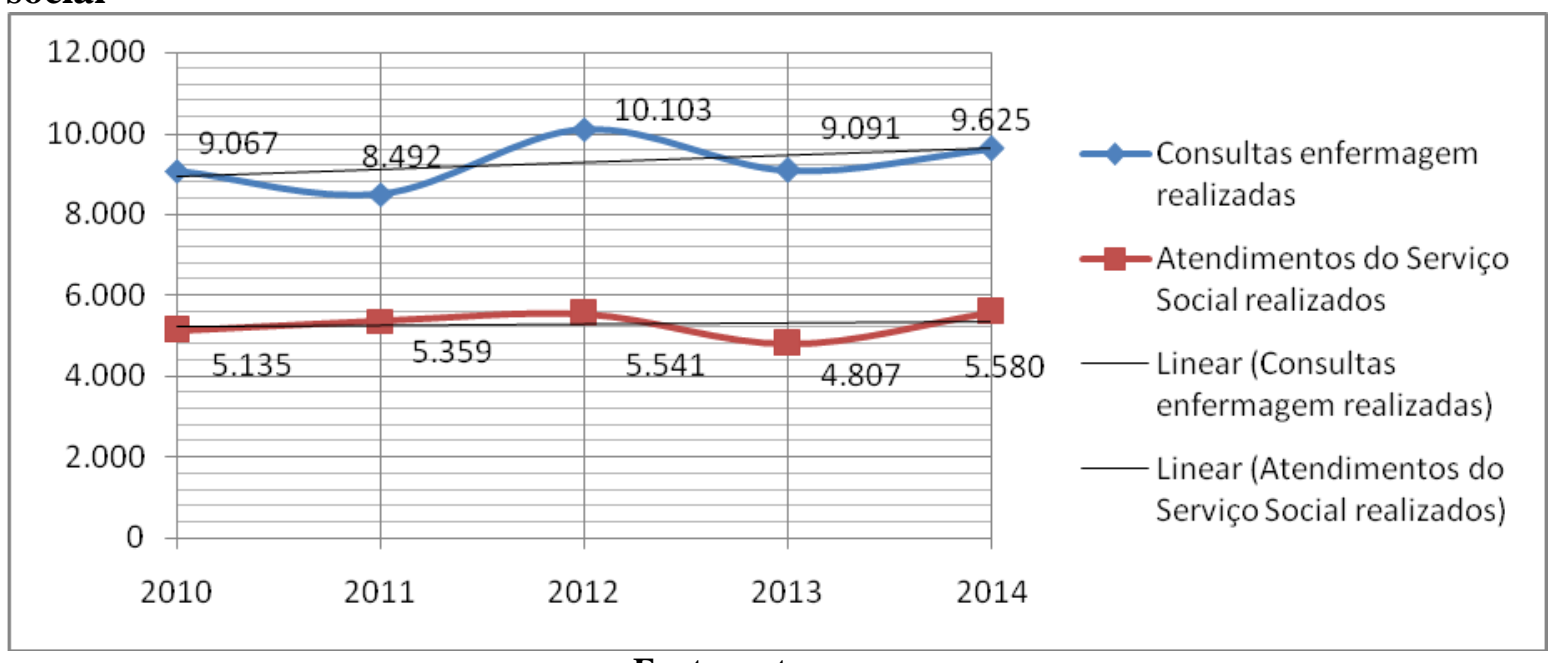

Fonte: autores.

As consultas de enfermagem realizadas neste período apresentaram uma pequena oscilação, com um aumento de $6,15 \%$, como podemos observar através da sua linha linear. A queda de aproximadamente 6,34\% observada em 2011 em relação ao ano de 2010, é justificada no relatorio de gestão de 2011 pela diminuição da carga horária de uma das enfermeiras, cedida à direção do CSVC até final de novembro daquele ano. Em 2012 há um aumento de 19\% nas consultas realizadas, apresentada no Relatório de Gestão como decorrência da demanda diária dos serviços, o que demandou do SAE IAPI a manutenção de uma enfermeira para atendimento exclusivo da demanda diária.

Já a redução de 10\% constatada no ano de 2013 se deve à Licença para Tratamento de Saúde de uma profissional do SAE CSVC e a licença prêmio de uma profissional do SAE IAPI, conforme o Relatório de Gestão correspondente (PORTO ALEGRE, 2013). Ao acompanharmos a evolução dos atendimentos de Serviço Social ao longo do período de 2010 à 2014, verificamos um acrescimo de aproximadamente $8,70 \%$. O Serviço Social do SAE/CSVC teve aumentada sua equipe de assistentes sociais durante o ano de 2012, resultando na elevação de aproximadamente 3,4\% de seus atendimentos na área. A diminuição de 13,25\% nos atendimentos em 2013 é resultante, segundo o que consta no Relatório de Gestão deste ano, de Licença Tratamento de Saúde de um profissional do Serviço Social do SAE CSVC. Em 2014 não há justificativas para o comportamento destes indicadores no Relatório Anual de Gestão. 


\section{Gráfico 5 - Evolução da distribuição de insumos.}

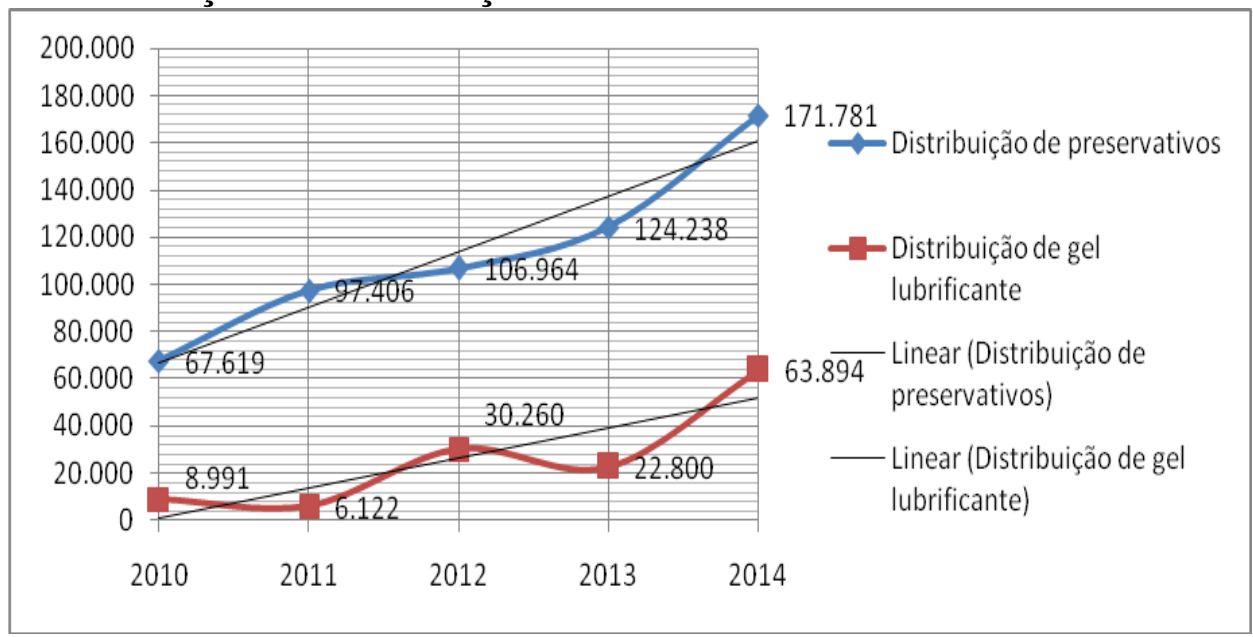

Fonte: autores.

A distribuição de insumos tem-se mantido em elevação no período analisado, conforme demonstra o gráfico 5. O número de preservativos distribuidos entre 2010 e 2014, totalizou um acrescimo de $155 \%$. Esse dado reflete a preocupação com ações de prevenção da doença e objetiva estabilizar as taxas de incidência que, conforme apontadas no quadro 1, tem sido bem elevadas no município desde 2007.

Em relação a distribuição de gel lubrificante, recurso auxiliar no uso do preservativo para impedir um possível rompimento do mesmo, assim como evitar as chances de sangramento e de transmissão de diversas doenças sexualmente transmissíveis, foram distribuídos 8.991 sachês no ano de 2010. É interessante ressaltar no entanto que este número foi corrigido no Relatório de Gestão do ano seguinte, onde passa a constar o número de 5.273 sachês distribuídos em 2010. No ano de 2011 a distribuição de gel lubrificante foi de 6.122 sachês. Assim, em decorrência da alteração nos dados do Relatório de Gestão de 2010, observa-se uma variação positiva de 13,86\% (e não uma queda de cerca de $30 \%$ se considerássemos os números anteriormente informados para o ano de 2010). Comparando o número total de gel lubrificante distribuído no período de 2010 a 2014, verifica-se um aumento de $611 \%$. 
Gráfico 6 - Evolução da taxa de incidência e coeficiente de mortalidade de HIV/AIDS no período de 2010 a 2014.

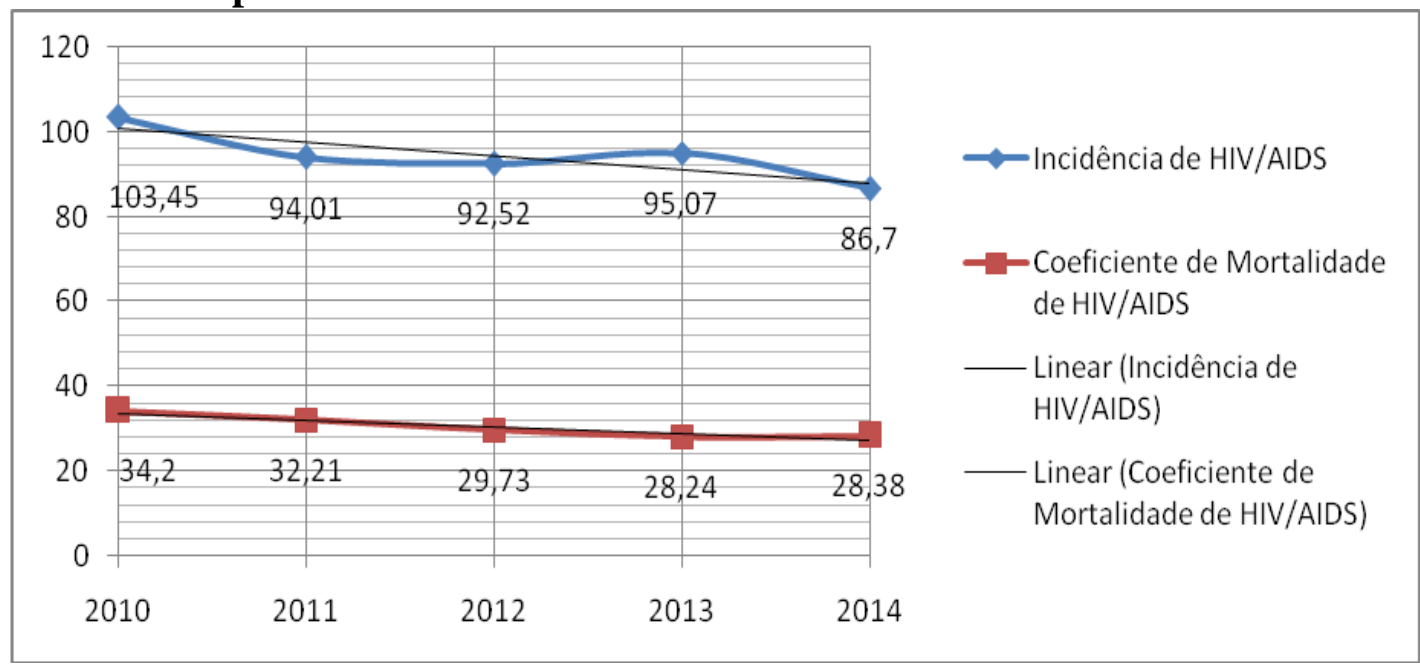

Fonte: autores.

Conforme o gráfico 6 constata-se a diminuição da taxa de incidência e na taxa de mortalidade no município Porta Alegre durante o período analisado. A taxa de incidência tem seu ápice em 2010, com 103,45 novos casos de HIV/AIDS no município. Em 2011 e 2012 houve, respectivamente, uma queda de 9,12\% e 1,59\% na taxa de incidência da doença. Este breve período de queda é alterado no ano de 2013 quando a taxa de incidência sobe para 95,07 casos por 100.000 habitantes, representando um acréscimo de $2,75 \%$ em relação ao ano anterior.

Os dados de 2014 apesar de sujeitos à revisão, como já informado anteriormente, apontam para a uma diminuição de 8,8\% em relação a 2013. Ao compararmos a evolução da taxa de incidência no ano de 2010 em relação ao ano de 2014, encontramos uma diminuição de aproximadamente 16,2\%. Quanto aos coeficientes de mortalidade de HIV/AIDS, se observa uma tendência de queda de aproximadamente $17 \%$ no período (2010 a 2014). No ano de 2014 houve um aumento de aproximadamente 0,5\%. A mortalidade por AIDS vem em queda gradativa desde a universalização da terapia antirretroviral. Entretanto, Porto Alegre ainda detém coeficientes de mortalidades elevadas, sendo que em 2013 apresentou 28,24 casos por 100.000 habitantes, mais que o dobro do RS, com 13,2 casos por 100.000 habitantes e 4,5 vezes maiores que a taxa nacional, de 6,4 casos por 100.000 habitantes (BRASIL, 2014).

O aumento significativo da distribuição dos preservativos, masculinos e femininos, assim como do gel lubrificante, parece ter influenciado na redução das taxas de incidência de HIV/AIDS no período. Esses dados chamam a atenção para a importância dos mesmos, o que fica evidenciado com o aumento significativo na sua 
distribuição em 2014. A diminuição de 27,6\% nas consultas médicas realizadas e $37,18 \%$ nas consultas disponibilizadas, ainda que decorrentes da falta de profissionais e licenças dos mesmos, não chegou a afetar as taxas de incidência e mortalidade conforme pudemos acompanhar, mantendo-se em declínio no período analisado. No entanto, podemos nos perguntar os motivos pelos quais, de modo recorrente, há uma significativa diferença entre as consultas disponibilizadas e as realizadas de fato. Tal situação pode indicar, entre outras coisas, uma dificuldade na adesão dos pacientes ao tratamento do HIV/AIDS ou mesmo a falta de acesso, questões que certamente merecem uma investigação mais apurada por outras pesquisas que venham a ser realizadas.

As consultas de enfermagem e os atendimentos de serviço social, apesar de algumas variações demonstradas, apresentaram uma tendência estável no período, demonstrando sua importância no controle da epidemia de HIV/AIDS e na diminuição das taxas de incidência e de mortalidade da doença, que apesar de ainda serem consideradas altas quando comparadas à outras cidades, apresentaram uma redução significativa.

\subsection{Aspectos qualitativos das ações e serviços em HIV/AIDS no município de Porto}

\section{Alegre/RS}

Para que seja possível a elaboração e implementação de políticas públicas relacionadas à epidemia de HIV/AIDS faz-se necessário, fundamentalmente, ter a compreensão dos chamados Determinantes Sociais de Saúde (DSS) para planejar e executar ações conjuntas e integradas entre os vários setores da sociedade. Essa afirmação, através da análise dos Relatórios, evidencia-se estar presente no modo como as ações e serviços de HIV/AIDS no município de Porto Alegre foram planejadas. Pela análise dos materiais, percebe-se que a gestão se fundamentou no modelo de compreensão sobre os determinantes sociais de saúde que se assemelham a aqueles propostos por Buss e Pellegrini Filho (2007). De acordo com o modelo proposto pelos autores, haveria quatro níveis de compreensão que permitem identificar pontos para intervenções no sentido de minimizar os diferenciais dos DSS originados pela posição social dos indivíduos e grupos: 
a) primeiro nível: os fatores comportamentais e os diferentes estilos de vida são extremamente influenciados pelos DSS, uma vez que mudar comportamentos de risco sem levar em conta as normas culturais que os influenciam é um desafio;

b) segundo nível: para a promoção e proteção da saúde individual e coletiva, é necessário incrementar os laços de entendimento social e as redes de solidariedade e confiança entre pessoas e grupos.

c) terceiro nível: reflete sobre as políticas das condições materiais e psicossociais. Ou seja, políticas públicas por melhores condições de habitação, de serviços de saúde e educação de qualidade.

d) quarto nível: compete as políticas macroeconômicas e de mercado de trabalho que visam à promoção de um desenvolvimento sustentável, para reduzir as desigualdades sociais econômicas (BUSS E PELLEGRINI FILHO, 2007p. 85).

Com base neste modelo de compreensão, a Secretaria Municipal de Saúde de Porto Alegre, em conjunto com a UNAIDS, desenvolveu um plano integrado de ações. Em maio de 2012 foi realizada uma importante reunião entre a as duas instituições com vistas à construção de um plano integrado de ações UNAIDS/Porto Alegre para o enfrentamento da epidemia do HIV/AIDS no município. Foram construídos e então apresentados nesta reunião os cinco eixos temáticos prioritários para o enfrentamento do HIV/AIDS pela Secretaria Municipal de Saúde de Porto Alegre, constantes na Proposta de Plano Integrado SMS UNAIDS - AIDS Tchê de 2012: eixo 1: Ampliação da cobertura de diagnóstico; b) eixo 2: Gestão da clínica; c) eixo 3: Promoção à saúde e prevenção de DST, HIV e AIDS; d) eixo 4: Comitê de Mortalidade por AIDS; e) eixo 5: Governança e fortalecimento dos movimentos sociais (PORTO ALEGRE, 2012).

Com base na análise dos materiais, infere-se que a proposta de plano integrado da SMS/PMPA mantêm conformidade com a primeira diretriz estratégica da UNAIDS (PROGRAMA CONJUNTO DAS NAÇÕES UNIDAS SOBRE HIV/AIDS, 2010) que tem como escopo gerar o compromisso político de atender às formas e às razões pelas quais os indivíduos acabam se infectando, mobilizando comunidades para que efetivamente exijam mudanças sociais e legais transformadoras, bem como para direcionar recursos para o foco de transmissão da epidemia por meio de intervenções corretas. De acordo com a Proposta de Plano Integrado SMS UNAIDS - AIDS Tchê de 2012, as estratégias de prevenção que deverão ser implementadas possuem metas e objetivos a serem alcançados em cinco anos, descritos a seguir: 
a) Observatório do HIV/AIDS: Acompanhar a evolução da epidemia em uma perspectiva interdisciplinar;

b) Prevenção do HIV/AIDS na população de jovens: Desenvolver ações de prevenção inseridas na Saúde e Prevenção nas Escolas;

c) Plano de enfrentamento da feminização da epidemia: Construir um plano de avaliação sobre as ações direcionadas às mulheres;

d) Ações preventivas junto à população negra: Estabelecer estratégias de combate ao racismo institucional;

e) Plano enfrentamento do HIV/AIDS na população de gays, e travestis e transexuais femininas: Conhecer os contextos de vulnerabilidades e cenários epidemiológicos;

f) Fortalecimento da intersetorialidade: Fortalecimento de ações intersetoriais no enfrentamento da epidemia do HIV/AIDS;

g) Fortalecimento do movimento social: Fortalecer a organização e participação de atores da sociedade civil organizada (PORTO ALEGRE, 2012, p.19-24).

A proposta de plano integrado aponta aspectos qualitativos que necessitam da articulação entre diversos atores da sociedade para que possam abordar a incidência de HIV/AIDS de forma diferenciada. Compreende-se que esta proposta exige um esforço coletivo e engajado de gestores, sociedade civil e agências internacionais de controle da epidemia para que se possa incidir de forma significativa e eficaz sobre o panorama atual do HIV/AIDS no município.

\section{Considerações finais}

As ações e serviços de saúde direcionadas ao enfrentamento do HIV/AIDS são fundamentais para o acompanhamento e qualificação da assistência aos portadores do vírus e para o controle e diminuição dos casos de infecção. Nesse artigo, a análise dos Relatórios de Gestão da SMS/PMPA permitiu evidenciar as ações e serviços de atenção especializada em HIV/AIDS no município de Porto Alegre e, assim, analisar o percurso dessas ações no período selecionado e o possível impacto das mesmas nas taxas de incidências e de mortalidade da doença.

A partir do ano de 2013, com as mudanças decorrentes das novas legislações, as informações constantes no Relatório Anual de Gestão passam a ser apresentadas de uma nova forma. Com a RENASES - Relação Nacional de Ações e Serviços de Saúde, de 
2014, por exemplo, a nomenclatura dos serviços de atenção ao HIV/AIDS, antes denominados como "serviços especializados", altera para "serviços de atenção especializada". Essa mudança, assim como as consultas médicas disponibilizadas, agendadas e realizadas, que a partir do Relatório Anual de Gestão de 2013 foram separadas em primeiras consultas e consultas de retorno, dificultaram a leitura e compreensão das informações disponíveis, exigindo atenção redobrada em sua análise. Assim também, os dados de 2013 tiveram que ser trabalhados em duas diferentes tabelas e, em 2014, em três diferentes tabelas, pois foi inaugurado um novo serviço, o SAE - Serviço de Atenção Especializada - Santa Marta.

Sugere-se que os dados poderiam ser organizados de maneira acessível a todo e qualquer cidadão, pois quando isso não acontece dificulta e limita o exercício da participação e controle social por parte da sociedade. Entende-se que as diferentes gestões, mudanças de governo e forças políticas podem atuar na forma com que esses dados são organizados, mas não devem ferir nos princípios da continuidade da administração pública e na transparência das informações disponíveis à consulta de forma livre e transparente. Isso evidencia que o modo de organizar e traduzir os dados impacta significativamente nas possibilidades de avaliação dos serviços de saúde.

Corroborado pelos dados encontrados, afirma-se a importância dos registros e informações em saúde através dos documentos utilizados nesta pesquisa (Relatórios de Gestão, Boletins Epidemiológicos) e a disponibilidade dos mesmos de forma transparente e pública nos sites de seus respectivos órgãos. É importante o detalhamento das informações disponíveis, mantendo a possibilidade de entendimento, análises, comparações e críticas. Quanto mais informações disponíveis, melhores serão as condições para as análises que podem ser feitas acerca do tema, já que possibilita a realização de estudos estatísticos mais complexos sobre o cenário, incluindo a importância de pensar estratégias de humanização e integralidade do cuidado, como afirmam estudos (MELLO, 2010; GUARESCHI et al., 2014). Os materiais analisados também possibilitaram verificar a relação entre as ações e serviços de atendimento especializado em HIV/AIDS prestados pela SMS/PMPA, e as taxas de incidência e de mortalidade. Verificou-se a importância das ações de caráter preventivo, tais como o a distribuição dos preservativos e do gel lubrificante, assim como a relevância das ações de educação em saúde para diminuição das taxas de incidência e mortalidade de HIV/AIDS. 
Assim, através dos dados do artigo, confirma-se a importância de aliar a abordagem quantitativa e qualitativa, principalmente para responder de modo mais significativo às complexidades identificadas no campo da saúde, onde diversos atores, interesses e determinantes, interagem dinamicamente. Ao analisar a evolução das consultas médicas, por exemplo, nota-se a necessidade do planejamento e de uma nova política de pessoal para a área da saúde que garantam a continuidade do atendimento aos usuários apesar dos afastamentos ou licenças de profissionais, bem como, apresentem mecanismos mais ágeis de reposição de pessoal, fazendo um uso interligado dos instrumentos desenvolvidos no processo de planejamento e gestão, para operacionalização integrada, solidária e sistêmica do SUS.

A partir dos dados, pode-se destacar o valor das ações de caráter preventivo e a importância de iniciativas de conscientização coletiva. Ressalta-se, também, a importância da continuidade e qualificação da oferta das ações e serviços já garantidos e a necessidade de um olhar cada vez mais transdisciplinar para que as taxas de incidência e coeficientes de mortalidade de HIV/AIDS no município de Porto Alegre/RS diminuam e a população atendida tenha cada vez mais disponíveis recursos de cuidado.

\section{REFERÊNCIAS}

BRASIL. Lei n ${ }^{\circ} 9.313$ de 13 de novembro de 1996. Dispõe sobre a distribuição gratuita de medicamentos aos portadores do HIV e doentes de AIDS[...]. Diário Oficial [da] República Federativa do Brasil. Brasília, DF. 1996. Disponível em: <http://www.planalto.gov.br/cci vil_03/leis/19313.htm> Acesso em: 30 jul. 2018.

BRASIL. Ministério do Planejamento, Orçamento e Gestão. Censo do ano de 2000. Instituto Brasileiro de Geografia e Estatística (IBGE). Brasília, DF. 2000.

BRASIL. Ministério da Saúde. Política Brasileira de Enfrentamento da AIDS: Resultados, Avanços e Perspectivas. Brasília, DF. 2012a.

BRASIL. Ministério da Saúde. Relação Nacional de Ações e Serviços de Saúde (RENASES). Brasília, DF, 2012b. Disponível em: <http://conitec.gov.br/images/Protocolos/Renases2012.pdf> Acesso em: 30 jul. 2012. 
BRASIL. Ministério da Saúde. Boletim Epidemiológico Aids e DST. Ano II, $\mathrm{N}^{\circ} 01$ Brasília, DF. 2013, p. 13. Disponível em: <http://www.aids.gov.br/ptbr/pub/2013/boletim-epidemiologico-hivaids-2013> Acesso em: 30 jul. 2018.

BRASIL. Ministério da Saúde. Boletim Epidemiológico Aids e DST. Ano III, No 01. Brasília, DF. 2014. Disponível em: <http://www.aids.gov.br/pt-br/node/73> Acesso em: 30 jul. 2018.

BRASIL. Ministério da Saúde. Boletim epidemiológico HIV/Aids 2017. Brasília, DF. 2017. Disponível em: <http://www.aids.gov.br/pt-br/pub/2017/boletim-epidemiologicohivaids-2017> Acesso em: 06 nov. 2018.

BRASIL. Perguntas e Respostas - Frequentes. Conselho Nacional de Saúde. Ministério da Saúde. Brasília, DF. 2018. Disponível em: <http://conselho.saude.gov.br/web_comissoes/conep/in dex.html> Acesso em: 30 jul. 2018.

DIEHL, A. A. Pesquisa em ciências sociais aplicadas: métodos e técnicas. São Paulo: Prentice Hall, 2004.

FONSECA, J. J. S. Metodologia da pesquisa científica. Fortaleza: UEC, 2002. Apostila.

GIL, A.C. Métodos e técnicas de pesquisa social. São Paulo: Atlas, 1991

GUARESCHI, N. M. de F et al. Formação em psicologia: o princípio da integralidade e a teoria da autopoiese. Rev. Psicol. Saúde, Campo Grande, v.6, n.1, p.18-27, jun. 2014. Disponível em <http://pepsic.bvsalud.org/scielo.php?script=sci_arttext\&pid=S2177093X2014000100004\&lng=pt \&nrm=iso>. Acessos em 30 jul. 2018.

LEVI, G. C; VITÓRIA, M. A. Fighting against AIDS: the Brazilian experience. Editorial Review, Vol.16, $\mathrm{N}^{\circ} 18,2002 . \quad$ Disponível em: <http://hspe.phlnet.com.br/phl8/tc/art33.pdf $>$ Acesso em: 30 jul. 2018.

MAYRING, P. H. Introdução à pesquisa social qualitativa. ( $5^{\mathrm{a}}$ ed.). Weinheim: Beltz, 2002.

MELLO, V. Estratégias de Humanização do cuidado em Saúde Mental: cartografando as intervenções de apoiadores institucionais. 2009. Dissertação (Mestrado em Psicologia) - Programa de Pós-Graduação em Psicologia Social e Institucional, Universidade Federal do Rio Grande do Sul, Porto Alegre. 2009. 
MINAYO, M. C. S. Pesquisa social: teoria, método e criatividade. Petrópolis: Vozes, 2001.

PEREIRA, S. D. Conceitos e Definições em Epidemiologia importantes para Vigilância Sanitária. $2004 . \quad$ Disponível em: <http://www.cvs.saude.sp.gov.br/pdf/epid_visa.pdf> Acesso em: 30 jul. 2018.

POPE, C.; MAYS, N. Pesquisa qualitativa na atenção à saúde. Artmed, 2009.

PORTO ALEGRE. Secretaria Municipal da Saúde. Plano Municipal de Saúde 20102013. Porto Alegre, RS. 2010. Disponível em: $<$ http://lproweb.procempa.com.br/pmpa/prefpoa/sms/usu_doc/pm s.pdf $>$ Acesso em: 30 jul. 2018.

PORTO ALEGRE. Secretaria Municipal de Saúde. Proposta de Plano Integrado SMS UNAIDS - AIDS Tchê. Área Técnica de DST/AIDS e Hep.Virais. Porto Alegre, RS. 2012.

PORTO ALEGRE. Secretaria Municipal da Saúde. Boletim Epidemiológico $\mathbf{n}^{\circ}$ 51, maio de 2013. Porto Alegre, RS. 2013.

PORTO ALEGRE. Secretaria Municipal da Saúde. Plano Municipal de Saúde 20142017. Porto Alegre, RS. 2014. Disponível em: <http://lproweb.procempa.com.br/pmpa/prefpoa/sms/usu_doc/pm s_2014_2017.pdf>. Acesso em: 30 jul. 2018.

PORTO ALEGRE. Prefeitura Municipal de Porto Alegre. Partenon - Lomba do Pinheiro. Porto Alegre, RS. 2015. Disponível em: $<$ http://www2.portoalegre.rs.gov.br/conferenciasaude/default.php ?psecao=23>. Acesso em: 06 nov. 2018.

PORTO ALEGRE. Secretaria Municipal da Saúde. Estrutura. Porto Alegre, RS. 2018. Disponível em: <http://www2.portoalegre.rs.gov.br/sms/default.php?p_secao=808>. Acesso em: 30 jul. 2018.

PORTO ALEGRE. Secretaria Municipal da Saúde. Relatório Anual de Gestão 2010. Porto Alegre, 2010.2 DS. 2 em: <http://lproweb.procempa.com.br/pmpa/prefpoa/sms/usudoc/ra g_20 10.pdf> Acesso em: 30 jul. 2018. 
PORTO ALEGRE. Secretaria Municipal da Saúde. Relatório Anual de Gestão 2011.

Porto Alegre, RS. 2011. Disponível em:

〈http://lproweb.procempa.com.br/pmpa/prefpoa/sms/usu_doc/rel

atorio_anual_de_gestao_2011.pdf $>$ Acesso em: 30 jul. 2018.

PORTO ALEGRE. Secretaria Municipal da Saúde. Relatório Anual de Gestão 2012.

Porto Alegre, $2012 . \quad$ DS. 2 em: $<$ http://lproweb.procempa.com.br/pmpa/prefpoa/sms/usu_doc/re

latorio_anual_de_gestao_2012.pdf> Acesso em: 30 jul. 2018.

PORTO ALEGRE. Secretaria Municipal da Saúde. Relatório Anual de Gestão 2013. Porto Alegre: $2013 . \quad$ RS. Disponível em: <http://lproweb.procempa.com.br/pmpa/prefpoa/sms/usu_doc/ra g_2013.pdf> Acesso em: 30 jul. 2018.

PORTO ALEGRE. Secretaria Municipal de Saúde. Relatório Anual de Gestão 2014. Porto Alegre, RS. 2014. Disponível em: <http://lproweb.procempa.com.br/pmpa/prefpoa/sms/usu_doc/rag_2014.pdf_>. Acesso em: 30 jul. 2018.

PROGRAMA CONJUNTO DAS NAÇÕES UNIDAS SOBRE HIV/AIDS. Chegando a zero: estratégia 2011-2015. UNAIDS/ONUSIDA, 2010. Disponível em: $<$ http://files.unaids.org/en/ media/unaids/contentassets/documents/unaidspublication/2010/JC2034_UNAIDS_Strat egy_pt.pdf> Acesso em: 30 jul. 2018.

RICHARDSON, R. J. Pesquisa social: métodos e técnicas. São Paulo: Atlas, 1989.

RIO GRANDE DO SUL. Secretaria Estadual da Saúde. Linha de Cuidado para Pessoas Vivendo com HIV/Aids (PVHA) e outras DST. 2014.

VOGT, W. P. Dictionary of statistics and methodology: A nontechnical guide for the social scientist. Newbury Park: Sage, 1993.

YIN, R. K. Estudo de caso: planejamento e métodos. Trad. de Daniel Grassi. Porto Alegre: Bookman, 2001. 